\title{
Hyers-Ulam stability of first-order linear differential equations using Aboodh transform
}

\author{
Ramdoss Murali ${ }^{1}$, Arumugam Ponmana Selvan², Sanmugam Baskaran', Choonkil Park ${ }^{3^{*}}$ (D) and Jung \\ Rye Lee ${ }^{4^{*}}$
}

"Correspondence:

baak@hanyang.ac.kr;

jrlee@daejin.ac.kr

${ }^{3}$ Research Institute for Natural Sciences, Hanyang University, 04763

Seoul, Korea

${ }^{4}$ Department of Data Science,

Daejin University, 11159 Kyunggi,

Korea

Full list of author information is

available at the end of the article

\section{Springer}

\begin{abstract}
The main aim of this paper is to investigate various types of Ulam stability and Mittag-Leffler stability of linear differential equations of first order with constant coefficients using the Aboodh transform method. We also obtain the Hyers-Ulam stability constants of these differential equations using the Aboodh transform and some examples to illustrate our main results are given.
\end{abstract}

MSC: Primary 34K20; 26D10; 39B82; secondary 44A10; 34A40; 39A30

Keywords: Hyers-Ulam stability; Mittag-Leffler-Hyers-Ulam-Rassias stability; Aboodh transform; Linear differential equation

\section{Introduction}

In 1940, Ulam [1] proposed the following stability problem: When is the statement of the theorem still true or nearly true, despite slight variations on the theorem's hypotheses? In the following year, Hyers [2] gave the first positive answer to Ulam's question by proving the stability of the additive functional equation in Banach spaces. Since then, Hyers' result has been widely generalized in terms of control conditions used to define the concept of an approximate solution (see [3-7]).

The generalization of Ulam's question has been relatively recently proposed by replacing functional equations with differential equations: Let $I$ be a subinterval of $\mathbf{R}$, let $\mathbf{K}$ denote either $\mathbf{R}$ or $\mathbf{C}$, and let $n$ be a positive integer. The differential equation $\psi\left(f, z, z^{\prime}, z^{\prime \prime}, \ldots, z^{(n)}\right)=$ 0 has the Hyers-Ulam stability if there exists a constant $K>0$ such that the following statement is true: for every $\varepsilon>0$, if an $n$ times continuously differentiable function $z: I \rightarrow \mathbf{K}$ satisfies the inequality

$$
\left|\psi\left(f, z, z^{\prime}, z^{\prime \prime}, \ldots, z^{(n)}\right)\right| \leq \varepsilon
$$

for all $t \in I$, then there exists a solution $y: I \rightarrow \mathbf{K}$ of the differential equation that satisfies the inequality $|z(t)-y(t)| \leq K \varepsilon$ for all $t \in I$.

(c) The Author(s) 2021. This article is licensed under a Creative Commons Attribution 4.0 International License, which permits use, sharing, adaptation, distribution and reproduction in any medium or format, as long as you give appropriate credit to the original author(s) and the source, provide a link to the Creative Commons licence, and indicate if changes were made. The images or other third party material in this article are included in the article's Creative Commons licence, unless indicated otherwise in a credit line to the material. If material is not included in the article's Creative Commons licence and your intended use is not permitted by statutory regulation or exceeds the permitted use, you will need to obtain permission directly from the copyright holder. To view a copy of this licence, visit http://creativecommons.org/licenses/by/4.0/. 
Obłoza seems to be the first author who investigated the Hyers-Ulam stability of linear differential equations (see [8,9]). Then, in 1998, Alsina and Ger [10] continued the study of Obłoza's Hyers-Ulam stability of differential equations. Indeed, they proved the following theorem.

Theorem 1.1 ([10]) Let $I \neq \emptyset$ be an open subinterval of $\mathbf{R}$. If a differentiable function $x$ : $I \rightarrow \mathbf{R}$ satisfies the differential inequality $\left\|x^{\prime}(t)-x(t)\right\| \leq \varepsilon$ for any $t \in I$ and for some $\varepsilon>0$, then there exists a differentiable function $y: I \rightarrow \mathbf{R}$ satisfying $y^{\prime}(t)=y(t)$ and $\|x(t)-y(t)\| \leq$ $3 \varepsilon$ for all $t \in I$.

This result of Alsina and Ger has been generalized by Takahashi et al. [11]. They proved that the Hyers-Ulam stability holds true for the Banach space valued differential equation $x^{\prime}(t)=\lambda x(t)$. Indeed, the Hyers-Ulam stability has been proved for the first-order linear differential equations in more general settings (see [12-18]).

In 2006, Jung [15] investigated the Hyers-Ulam stability of a system of first-order linear differential equations with constant coefficients by using matrix method. Then, in 2008, Wang et al. [19] studied the Hyers-Ulam stability of linear differential equations of first order using the integral factor method. Meanwhile, Rus [20] discussed various types of Hyers-Ulam stability of the ordinary differential equation of the form $x^{\prime}(t)=$ $A x(t)+f(t, x(t))$.

In 2014, Alqifiary and Jung [21] proved the Hyers-Ulam stability of linear differential equation of the form

$$
x^{(n)}(t)+\sum_{k=0}^{n-1} \alpha_{k} x^{(k)}(t)=f(t)
$$

by using the Laplace transform method, where $\alpha_{k}$ are scalars and $x(t)$ is an $n$ times continuously differentiable function and of exponential order (see also [22, 23]).

In recent years, many authors are studying the Hyers-Ulam stability of differential equations, and a number of mathematicians are paying attention to new results of the HyersUlam stability of differential equations (see [24-37]). Recently, Murali et al. [38] have investigated the Hyers-Ulam stability of the linear differential equation using Fourier transform method (see also [39, 40]).

Very recently, Murali and Ponmana Selvan [41] studied the Mittag-Leffler-Hyers-Ulam stability of a linear differential equations of first order using Laplace transforms method.

Integral transform plays an important role in solving ordinary differential equation. The Laplace transform has been effectively used to solve linear and nonlinear ordinary and partial differential equations and it is used extensively in proving Ulam stability problem.

Aboodh integral transform [42, 43] was introduced by Aboodh in the year 2013, to facilitate the process of solving ordinary differential equations in the time domain. This transformation has deeper connection with the Laplace and Elzaki transform.

Based on the above results, our main intention is to more efficiently prove the HyersUlam stability and Mittag-Leffler-Hyers-Ulam stability of the first-order linear differential equations of the forms

$$
x^{\prime}(t)+\lambda x(t)=0
$$


and

$$
x^{\prime}(t)+\lambda x(t)=r(t)
$$

with the help of the Aboodh transform method, where $\lambda$ is a scalar and $x(t)$ is a continuously differentiable function of exponential order.

\section{Preliminaries and basic notations}

In this section, we introduce some standard notations and definitions which will be useful to prove our main results.

Throughout this paper, $\mathbf{K}$ denotes either the real field $\mathbf{R}$ or the complex field $\mathbf{C}$. A function $f:[0, \infty) \rightarrow \mathbf{K}$ is of exponential order if there exist constants $A, B \in \mathbf{R}$ such that $|f(t)| \leq A e^{B t}$ for all $t \geq 0$.

Consider the set $S$, which is defined by

$$
S=\left\{f:[0, \infty) \rightarrow \mathbf{K} / \exists M, k_{1}, k_{2}>0,|f(t)|<M e^{-u t}, \forall u \in\left(k_{1}, k_{2}\right), \forall t \in[0, \infty)\right\}
$$

For a given function $f(t)$ in the set $S, M$ must be a finite number, $k_{1}$ and $k_{2}$ may be finite or infinite [44]. Now, we define the Aboodh transform as follows.

Definition 2.1 ([44, 45]) The Aboodh (integral) transform is defined, for a function $f(t)$ of exponential order, by

$$
\mathcal{A}\{f(t)\}=\frac{1}{u} \int_{0}^{\infty} f(t) e^{-u t} d t=F(u), \quad t \geq 0
$$

provided that the integral exists for some $u$, where $u \in\left(k_{1}, k_{2}\right)$. Here $\mathcal{A}$ is called the Aboodh (integral) transform operator.

The Aboodh integral transform for a function $f:[0, \infty) \rightarrow \mathbf{K}$ exists if $f(t)$ is piecewise continuous and of exponential order. These conditions are the only sufficient conditions for the existence of the Aboodh transform of a function $f(t)$ (see [44-46]).

Definition 2.2 ([47] (Convolution of two functions)) The convolution of two functions $f(t)$ and $g(t)$ is denoted by $f(t) * g(t)$ and is defined by

$$
f(t) * g(t)=(f * g)(t)=\int_{0}^{t} f(s) g(t-s) d s=\int_{0}^{t} f(t-s) g(s) d s .
$$

Theorem 2.3 ([47] (Convolution theorem for Aboodh transform)) Assume that $f(t)$ and $g(t)$ are given functions defined for $t \geq 0$. If $\mathcal{A}\{f(t)\}=F(u)$ and $\mathcal{A}\{g(t)\}=G(u)$, then

$$
\mathcal{A}\{f(t) * g(t)\}=u \mathcal{A}\{f(t)\} \mathcal{A}\{g(t)\}=u F(u) G(u) .
$$

Definition 2.4 ([47] (Inverse Aboodh transform)) If $\mathcal{A}\{f(t)\}=F(u)$, then $f(t)$ is called the inverse Aboodh (integral) transform of $F(u)$ and is denoted as $f(t)=\mathcal{A}^{-1}\{F(u)\}$, where $\mathcal{A}^{-1}$ is the inverse Aboodh transform operator. 
Definition 2.5 ([48]) The Mittag-Leffler function of one parameter is denoted by $E_{v}(t)$ and it is defined as

$$
E_{v}(t)=\sum_{k=0}^{\infty} \frac{t^{k}}{\Gamma(v k+1)},
$$

where $t, v \in \mathbf{C}$ and $\Re(v)>0$. If we put $v=1$, then the above equation becomes

$$
E_{1}(t)=\sum_{k=0}^{\infty} \frac{t^{k}}{\Gamma(k+1)}=\sum_{k=0}^{\infty} \frac{t^{k}}{k !}=e^{t}
$$

Definition $2.6([48])$ The generalization of $E_{v}(t)$ is defined as a function

$$
E_{v, \vartheta}(t)=\sum_{k=0}^{\infty} \frac{t^{k}}{\Gamma(v k+\vartheta)}
$$

where $t, v, \vartheta \in \mathbf{C}, \Re(v)>0$ and $\Re(\vartheta)>0$.

Now we give the definitions of the Hyers-Ulam stability and the Hyers-Ulam $\phi$-stability of the differential equations (1.1) and (1.2).

Throughout this section, we set

$$
\mathcal{F}:=\{f:[0, \infty) \rightarrow \mathbf{K} \mid
$$

$f$ is a continuously differentiable function of exponential order $\}$.

Definition 2.7 The linear differential equation (1.1) is said to have the Hyers-Ulam stability (for the class $\mathcal{F}$ ) when there exists a constant $K>0$ such that the following statement is true for any $\varepsilon>0$ : If a function $x \in \mathcal{F}$ satisfies the inequality

$$
\left|x^{\prime}(t)+\lambda x(t)\right| \leq \varepsilon
$$

for all $t \geq 0$, then there exists a solution $y:[0, \infty) \rightarrow \mathbf{K}$ of differential equation (1.1) such that $y \in \mathcal{F}$ and

$$
|x(t)-y(t)| \leq K \varepsilon
$$

for all $t \geq 0$.

Definition 2.8 We say that the non-homogeneous linear differential equation (1.2) has the Hyers-Ulam stability (for the class $\mathcal{F}$ ), if there exists a constant $K>0$ such that the following statement is true for each $\varepsilon>0$ : If a function $x \in \mathcal{F}$ satisfies the inequality

$$
\left|x^{\prime}(t)+\lambda x(t)-r(t)\right| \leq \varepsilon
$$

for all $t \geq 0$, then there exists a solution $y:[0, \infty) \rightarrow \mathbf{K}$ of differential equation (1.2) such that $y \in \mathcal{F}$ and

$$
|x(t)-y(t)| \leq K \varepsilon
$$

for any $t \geq 0$. Then the constant $K$ is called a Hyers-Ulam constant. 
Definition 2.9 Let $\phi:[0, \infty) \rightarrow(0, \infty)$ be a function. We say that the homogeneous linear differential equation (1.1) has the Hyers-Ulam $\phi$-stability (for the class $\mathcal{F}$ ), if there exists a constant $K>0$ such that the following statement is true for every $\varepsilon>0$ : If a function $x \in \mathcal{F}$ satisfies the inequality

$$
\left|x^{\prime}(t)+\lambda x(t)\right| \leq \phi(t) \varepsilon
$$

for any $t \geq 0$, then there exists a solution $y:[0, \infty) \rightarrow \mathbf{K}$ of differential equation (1.1) such that $y \in \mathcal{F}$ and

$$
|x(t)-y(t)| \leq K \phi(t) \varepsilon
$$

for any $t \geq 0$.

Definition 2.10 Let $\phi:[0, \infty) \rightarrow(0, \infty)$ be a function. The differential equation (1.2) is said to have the Hyers-Ulam $\phi$-stability (for the class $\mathcal{F}$ ) when there exists a constant $K>0$ such that the following statement is true for all $\varepsilon>0$ : If a function $x \in \mathcal{F}$ satisfies the inequality

$$
\left|x^{\prime}(t)+\lambda x(t)-r(t)\right| \leq \phi(t) \varepsilon
$$

for all $t \geq 0$, then there exists a solution $y:[0, \infty) \rightarrow \mathbf{K}$ of differential equation (1.2) such that $y \in \mathcal{F}$ and

$$
|x(t)-y(t)| \leq K \phi(t) \varepsilon
$$

for all $t \geq 0$. For the case, we call the constant $K$ a Hyers-Ulam $\phi$-constant.

Finally, we introduce the definitions of Mittag-Leffler-Hyers-Ulam stability and MittagLeffler-Hyers-Ulam $\phi$-stability of the differential equations (1.1) and (1.2).

Definition 2.11 Let $E_{v}(t)$ be the Mittag-Leffler function. We say that the differential equation (1.1) has the Mittag-Leffler-Hyers-Ulam stability (for the class $\mathcal{F}$ ), if there exists a constant $K>0$ such that the following statement holds true for any $\varepsilon>0$ : If a function $x \in \mathcal{F}$ satisfies the inequality

$$
\left|x^{\prime}(t)+\lambda x(t)\right| \leq \varepsilon E_{v}(t)
$$

for all $t \geq 0$, then there exists a solution $y:[0, \infty) \rightarrow \mathbf{K}$ of differential equation (1.1) such that $y \in \mathcal{F}$ and

$$
|x(t)-y(t)| \leq K \varepsilon E_{v}(t)
$$

for any $t \geq 0$.

Definition 2.12 Let $E_{v}(t)$ be the Mittag-Leffler function. We say that the non-homogeneous differential equation (1.2) has the Mittag-Leffler-Hyers-Ulam stability (for the 
class $\mathcal{F}$ ) when there exists a constant $K>0$ such that the following statement is true for each $\varepsilon>0$ : If a function $x \in \mathcal{F}$ satisfies the inequality

$$
\left|x^{\prime}(t)+\lambda x(t)-r(t)\right| \leq \varepsilon E_{v}(t)
$$

for every $t \geq 0$, then there exists a solution $y:[0, \infty) \rightarrow \mathbf{K}$ of differential equation (1.2) such that $y \in \mathcal{F}$ and

$$
|x(t)-y(t)| \leq K \varepsilon E_{v}(t)
$$

for any $t \geq 0$. We call the constant $K$ a Mittag-Leffler-Hyers-Ulam constant.

Definition 2.13 Let $E_{v}(t)$ be the Mittag-Leffler function and let $\phi:[0, \infty) \rightarrow(0, \infty)$ be a function. We say that the differential equation (1.1) has the Mittag-Leffler-Hyers-Ulam $\phi$ stability (for the class $\mathcal{F}$ ), if there exists a constant $K>0$ such that the following statement is true for any $\varepsilon>0$ : If a function $x \in \mathcal{F}$ satisfies the inequality

$$
\left|x^{\prime}(t)+\lambda x(t)\right| \leq \phi(t) \varepsilon E_{v}(t)
$$

for each $t \geq 0$, then there exists a solution $y:[0, \infty) \rightarrow \mathbf{K}$ of differential equation (1.1) such that $y \in \mathcal{F}$ and

$$
|x(t)-y(t)| \leq K \phi(t) \varepsilon E_{v}(t)
$$

for any $t \geq 0$.

Definition 2.14 Let $E_{v}(t)$ be the Mittag-Leffler function and let $\phi:[0, \infty) \rightarrow(0, \infty)$ be a function. We say that the differential equation (1.2) has the Mittag-Leffler-Hyers-Ulam $\phi$-stability (for the class $\mathcal{F}$ ) when there exists a constant $K>0$ such that the following statement is true for any $\varepsilon>0$ : If a function $x \in \mathcal{F}$ satisfies the inequality

$$
\left|x^{\prime}(t)+\lambda x(t)-r(t)\right| \leq \phi(t) \varepsilon E_{v}(t)
$$

for any $t \geq 0$, then there exists a solution $y:[0, \infty) \rightarrow \mathbf{K}$ of differential equation (1.2) such that $y \in \mathcal{F}$ and

$$
|x(t)-y(t)| \leq K \phi(t) \varepsilon E_{v}(t)
$$

for any $t \geq 0$. For this case, we call $K$ a Mittag-Leffler-Hyers-Ulam $\phi$-constant.

If, in addition, minimum of such K's exists, then we call it as the best Ulam constant.

\section{Hyers-Ulam stability of (1.1)}

In this section, we prove several types of Hyers-Ulam stability of the homogeneous firstorder linear differential equation (1.1) using the Aboodh transform.

It should be noted that in this and the next sections we investigate various types of Hyers-Ulam stability in the class of continuously differentiable functions of exponential order. 
Theorem 3.1 Assume that $\lambda$ is a constant with $\Re(\lambda)>0$. The differential equation (1.1) is Hyers-Ulam stable in the class of continuously differentiable functions of exponential order.

Proof Assume that $x:[0, \infty) \rightarrow \mathbf{K}$ is a continuously differentiable function of exponential order which satisfies the inequality (2.1) for all $t \geq 0$. Let us define a function $p:[0, \infty) \rightarrow$ K by $p(t):=x^{\prime}(t)+\lambda x(t)$ for all $t \geq 0$. In view of (2.1), the inequality $|p(t)| \leq \varepsilon$ holds for all $t \geq 0$. The Aboodh transform of $p(t)$ gives the following result:

$$
P(u):=\mathcal{A}\{p(t)\}=\mathcal{A}\left\{x^{\prime}(t)+\lambda x(t)\right\}=\mathcal{A}\left\{x^{\prime}(t)\right\}+\lambda \mathcal{A}\{x(t)\}=u X(u)-\frac{x(0)}{u}+\lambda X(u),
$$

since $\mathcal{A}\left\{x^{\prime}(t)\right\}=u \mathcal{A}\{x(t)\}-\frac{x(0)}{u}$. Thus

$$
\mathcal{A}\{x(t)\}=X(u)=\frac{\frac{x(0)}{u}+P(u)}{\lambda+u} .
$$

If we put $y(t)=e^{-\lambda t} x(0)$, then $y(0)=x(0)$ and $y(t)$ is a function of exponential order. The Aboodh transform of $y(t)$ gives the following result:

$$
\mathcal{A}\{y(t)\}=Y(u)=\frac{x(0)}{u(\lambda+u)} .
$$

Thus,

$$
\mathcal{A}\left\{y^{\prime}(t)+\lambda y(t)\right\}=\mathcal{A}\left\{y^{\prime}(t)\right\}+\lambda \mathcal{A}\{y(t)\}=u Y(u)-\frac{y(0)}{u}+\lambda Y(u) .
$$

Using (3.2), we have $\mathcal{A}\left\{y^{\prime}(t)+\lambda y(t)\right\}=0$. Since $\mathcal{A}$ is a one-to-one operator, $y^{\prime}(t)+\lambda y(t)=0$. Hence $y(t)$ is a solution of the differential equation (1.1). By (3.1) and (3.2), we obtain

$$
\mathcal{A}\{x(t)\}-\mathcal{A}\{y(t)\}=X(u)-Y(u)=\frac{P(u)}{\lambda+u}=u P(u) Q(u)=\mathcal{A}\{p(t) * q(t)\},
$$

where $Q(u)=\frac{1}{u(\lambda+u)}$, which gives $q(t)=\mathcal{A}^{-1}\left\{\frac{1}{\lambda u+u^{2}}\right\}=e^{-\lambda t}$.

Consequently, $\mathcal{A}\{x(t)-y(t)\}=\mathcal{A}\{p(t) * q(t)\}$ and thus, $x(t)-y(t)=p(t) * q(t)$. Taking the modulus on both sides, we have

$$
\begin{aligned}
|x(t)-y(t)| & =|p(t) * q(t)|=\left|\int_{0}^{t} p(s) q(t-s) d s\right| \leq \int_{0}^{t}|p(s)||q(t-s)| d s \\
& \leq \varepsilon \int_{0}^{t}|q(t-s)| d s=\varepsilon e^{-\Re(\lambda) t} \int_{0}^{t} e^{\Re(\lambda) s} d s=\frac{\varepsilon}{\Re(\lambda)}\left(1-e^{-\Re(\lambda) t}\right) \\
& \leq K \varepsilon
\end{aligned}
$$

for all $t \geq 0$, where we set $K=\frac{1}{\Re(\lambda)}$, which implies that the differential equation (1.1) has the Hyers-Ulam stability.

Similar to Theorem 3.1, we will prove the Hyers-Ulam $\phi$-stability for the differential equation (1.1). For the sake of the completeness of this paper, the proof is introduced here in detail. 
Theorem 3.2 Assume that $\phi:[0, \infty) \rightarrow(0, \infty)$ is an increasing function and $\lambda$ is a constant with $\Re(\lambda)>0$. Then the differential equation (1.1) has the Hyers-Ulam $\phi$-stability.

Proof Let $x:[0, \infty) \rightarrow \mathbf{K}$ be a continuously differentiable function of exponential order and $\phi:[0, \infty) \rightarrow(0, \infty)$ be an increasing function satisfying the inequality (2.3) for all $t \geq 0$. If we define a function $p:[0, \infty) \rightarrow \mathbf{K}$ by $p(t):=x^{\prime}(t)+\lambda x(t)$ for all $t \geq 0$, then we have $|p(t)| \leq \phi(t) \varepsilon$ for all $t \geq 0$.

As we did in the first part of the proof of Theorem 3.1, we can prove that $y(t)=e^{-\lambda t} x(0)$ is a solution of the differential equation (1.1). On the other hand, $Q(u)=\frac{1}{u(\lambda+u)}$ shows that $q(t)=\mathcal{A}^{-1}\left\{\frac{1}{u(\lambda+u)}\right\}=e^{-\lambda t}$.

Moreover, it follows from (3.1) and (3.2) that

$$
\begin{aligned}
\mathcal{A}\{x(t)\}-\mathcal{A}\{y(t)\} & =X(u)-Y(u)=\frac{P(u)}{\lambda+u}=u P(u) Q(u) \\
& =u \mathcal{A}\{p(t)\} \mathcal{A}\{q(t)\}=\mathcal{A}\{p(t) * q(t)\},
\end{aligned}
$$

which yields $\mathcal{A}\{x(t)-y(t)\}=\mathcal{A}\left\{p(t) * e^{-\lambda t}\right\}$. Therefore, $x(t)-y(t)=p(t) * e^{-\lambda t}$.

Similar to the proof of Theorem 3.1, we can show that

$$
\begin{aligned}
|x(t)-y(t)| & =\left|p(t) * e^{-\lambda t}\right|=\left|\int_{0}^{t} p(s) e^{-\lambda(t-s)} d s\right| \leq \int_{0}^{t}|p(s)|\left|e^{-\lambda(t-s)}\right| d s \\
& \leq \phi(t) \varepsilon e^{-\Re(\lambda) t} \int_{0}^{t} e^{\Re(\lambda) s} d s=\frac{\phi(t) \varepsilon}{\Re(\lambda)}\left(1-e^{-\Re(\lambda) t}\right) \\
& \leq K \phi(t) \varepsilon
\end{aligned}
$$

for all $t \geq 0$, where we set $K=\frac{1}{\Re(\lambda)}$. This completes the proof.

Now, we are going to establish the Mittag-Leffler-Hyers-Ulam stability of differential equation (1.1) using the Aboodh transform.

Theorem 3.3 Let $\lambda$ and $v$ be constants satisfying $\Re(\lambda)>0$ and $v>0$. Then the differential equation (1.1) has the Mittag-Leffler-Hyers-Ulam stability.

Proof Suppose that $x:[0, \infty) \rightarrow \mathbf{K}$ is a continuously differentiable function of exponential order which satisfies the inequality (2.5) for all $t \geq 0$. Let $p:[0, \infty) \rightarrow \mathbf{K}$ be a function defined by $p(t):=x^{\prime}(t)+\lambda x(t)$ for all $t \geq 0$. In view of (2.5), we have $|p(t)| \leq \varepsilon E_{v}(t)$ for all $t \geq 0$.

The Aboodh transform of $p(t)$ yields the following result:

$$
P(u):=\mathcal{A}\{p(t)\}=\mathcal{A}\left\{x^{\prime}(t)+\lambda x(t)\right\}=u X(u)-\frac{x(0)}{u}+\lambda X(u) .
$$

Thus, we get

$$
\mathcal{A}\{x(t)\}=X(u)=\frac{x(0)+u P(u)}{\lambda u+u^{2}} .
$$


If we put $y(t)=e^{-\lambda t} x(0)$, then $y(0)=x(0)$ and $y(t)$ is a function of exponential order. Moreover, the Aboodh transform of $y(t)$ yields

$$
\mathcal{A}\{y(t)\}=Y(u)=\frac{x(0)}{u^{2}+\lambda u} .
$$

It follows from (3.4) that

$$
\begin{aligned}
\mathcal{A}\left\{y^{\prime}(t)+\lambda y(t)\right\} & =\mathcal{A}\left\{y^{\prime}(t)\right\}+\lambda \mathcal{A}\{y(t)\} \\
& =u Y(u)-\frac{y(0)}{u}+\lambda Y(u)=0 .
\end{aligned}
$$

Since $\mathcal{A}$ is a one-to-one operator, $y^{\prime}(t)+\lambda y(t)=0$. Hence $y(t)$ is a solution of the differential equation (1.1). If we set $Q(u)=\frac{1}{\lambda u+u^{2}}$, then the equality $\mathcal{A}\{q(t)\}=\frac{1}{(\lambda+u) u}$ implies that $q(t)=$ $e^{-\lambda t}$. By (3.3) and (3.4), we obtain

$$
\mathcal{A}\{x(t)\}-\mathcal{A}\{y(t)\}=X(u)-Y(u)=\frac{P(u)}{\lambda+u}=u P(u) Q(u) .
$$

Consequently, $\mathcal{A}\{x(t)-y(t)\}=\mathcal{A}\left\{p(t) * e^{-\lambda t}\right\}$, which gives $x(t)-y(t)=p(t) * e^{-\lambda t}$. Taking modulus on both sides and using the fact that $|p(t)| \leq \varepsilon E_{v}(t)$ for all $t \geq 0$ and since $E_{v}(t)$ is increasing for $t \geq 0$, we have

$$
\begin{aligned}
|x(t)-y(t)| & =\left|p(t) * e^{-\lambda t}\right|=\left|\int_{0}^{t} p(s) e^{-\lambda(t-s)} d s\right| \leq \int_{0}^{t}|p(s)|\left|e^{-\lambda(t-s)}\right| d s \\
& \leq \varepsilon E_{\nu}(t) e^{-\Re(\lambda) t} \int_{0}^{t} e^{\Re(\lambda) s} d s=\varepsilon E_{v}(t) \frac{1}{\Re(\lambda)}\left(1-e^{-\Re(\lambda) t}\right) \\
& =K \varepsilon E_{v}(t)
\end{aligned}
$$

for all $t \geq 0$, where we choose $K=\frac{1}{\Re(\lambda)}$. Then, referring to Definition 2.11, we can confirm that the differential equation (1.1) has the Mittag-Leffler-Hyers-Ulam stability.

Similar to the case of Theorem 3.3, the Mittag-Leffler-Hyers-Ulam $\phi$-stability of the differential equation (1.1) can be proved. For the sake of this paper's completeness, we present the whole proof.

Theorem 3.4 Assume that $\phi:[0, \infty) \rightarrow(0, \infty)$ is an increasing function and that $\lambda$ and $v$ are constants which satisfy $\Re(\lambda)>0$ and $v>0$. Then the differential equation (1.1) has the Mittag-Leffler-Hyers-Ulam $\phi$-stability.

Proof Let $x:[0, \infty) \rightarrow \mathbf{K}$ be a continuously differentiable function of exponential order and $\phi:[0, \infty) \rightarrow(0, \infty)$ be a function that satisfies the inequality (2.7) for all $t \geq 0$. We will prove that there exist a positive constant $K>0$ (independent of $\varepsilon$ ) and a solution $y:[0, \infty) \rightarrow \mathbf{K}$ of the differential equation (1.1) such that

$$
|x(t)-y(t)| \leq K \phi(t) \varepsilon E_{\nu}(t)
$$

for all $t \geq 0$. 
If we define a function $p:[0, \infty) \rightarrow \mathbf{K}$ by $p(t):=x^{\prime}(t)+\lambda x(t)$ for all $t \geq 0$, then we have $|p(t)| \leq \phi(t) \varepsilon E_{v}(t)$ for all $t \geq 0$. Then by applying the same method as in the proof of Theorem 3.3 , we can easily get

$$
\begin{aligned}
|x(t)-y(t)| & =\left|p(t) * e^{-\lambda t}\right|=\left|\int_{0}^{t} p(s) e^{-\lambda(t-s)} d s\right| \leq \int_{0}^{t}|p(s)|\left|e^{-\lambda(t-s)}\right| d s \\
& \leq \phi(t) \varepsilon E_{v}(t) e^{-\Re(\lambda) t} \int_{0}^{t} e^{\Re(\lambda) s} d s=\phi(t) \varepsilon E_{v}(t) \frac{1}{\Re(\lambda)}\left(1-e^{-\Re(\lambda) t}\right) \\
& \leq K \phi(t) \varepsilon E_{v}(t)
\end{aligned}
$$

for all $t \geq 0$, where we set $K=\frac{1}{\Re(\lambda)}$. Then, referring to Definition 2.13, we confirm that the differential equation (1.1) has the Mittag-Leffler-Hyers-Ulam $\phi$-stability.

\section{Hyers-Ulam stability of (1.2)}

In this section, we prove several types of Hyers-Ulam stability of the homogeneous firstorder linear differential equation (1.2) using the Aboodh transform.

Theorem 4.1 Assume that $r:[0, \infty) \rightarrow \mathbf{K}$ is a continuous function of exponential order and $\lambda$ is a constant with $\mathfrak{R}(\lambda)>0$. The differential equation (1.2) has the Hyers-Ulam stability.

Proof Suppose that $x:[0, \infty) \rightarrow \mathbf{K}$ is a continuously differentiable function of exponential order which satisfies the inequality (2.2) for all $t \geq 0$. Consider the function $p:[0, \infty) \rightarrow \mathbf{K}$ defined by

$$
p(t):=x^{\prime}(t)+\lambda x(t)-r(t)
$$

for all $t \geq 0$. Then $|p(t)| \leq \varepsilon$ for all $t \geq 0$. The Aboodh transform of $p(t)$ gives the following result: $\mathcal{A}\{p(t)\}=\mathcal{A}\left\{x^{\prime}(t)+\lambda x(t)-r(t)\right\}$. That is,

$$
P(u):=\mathcal{A}\left\{x^{\prime}(t)\right\}+\lambda \mathcal{A}\{x(t)\}-\mathcal{A}\{r(t)\}=u X(u)-\frac{x(0)}{u}+\lambda X(u)-R(u),
$$

which implies that

$$
\mathcal{A}\{x(t)\}=X(u)=\frac{x(0)+u P(u)+u R(u)}{u(\lambda+u)} .
$$

We set $y(t)=e^{-\lambda t} x(0)+\left(r(t) * e^{-\lambda t}\right)$. The Aboodh transform of $y(t)$ yields the following result:

$$
\mathcal{A}\{y(t)\}=Y(u)=\frac{x(0)+u R(u)}{u(\lambda+u)} .
$$

On the other hand,

$$
\mathcal{A}\left\{y^{\prime}(t)+\lambda y(t)\right\}=u Y(u)-\frac{y(0)}{u}+\lambda Y(u)=(\lambda+u) Y(u)-\frac{x(0)}{u},
$$


since $x(0)=y(0)$. Then, by $(4.2)$, we have

$$
\mathcal{A}\left\{y^{\prime}(t)+\lambda y(t)\right\}=R(u)=\mathcal{A}\{r(t)\}
$$

and thus, $y^{\prime}(t)+\lambda y(t)=r(t)$. Hence $y(t)$ is a solution of the differential equation (1.2).

In addition, by applying (4.1) and (4.2), we can obtain

$$
\mathcal{A}\{x(t)\}-\mathcal{A}\{y(t)\}=X(u)-Y(u)=\frac{P(u)}{\lambda+u}=u P(u) Q(u)=u \mathcal{A}\{p(t)\} \mathcal{A}\{q(t)\},
$$

where we set $Q(u)=\frac{1}{u \lambda+u^{2}}$ which gives $q(t)=\mathcal{A}^{-1}\left\{\frac{1}{u \lambda+u^{2}}\right\}=e^{-\lambda t}$. Therefore, we have

$$
\mathcal{A}\{x(t)-y(t)\}=\mathcal{A}\left\{p(t) * e^{-\lambda t}\right\},
$$

which yields $x(t)-y(t)=p(t) * e^{-\lambda t}$. Furthermore,

$$
\begin{aligned}
|x(t)-y(t)| & =\left|p(t) * e^{-\lambda t}\right|=\left|\int_{0}^{t} p(s) e^{-\lambda(t-s)} d s\right| \leq \int_{0}^{t}|p(s)|\left|e^{-\lambda(t-s)}\right| d s \\
& \leq \varepsilon e^{-\Re(\lambda) t} \int_{0}^{t} e^{\Re(\lambda) s} d s \leq K \varepsilon
\end{aligned}
$$

for all $t \geq 0$, where we set $K=\frac{1}{\Re(\lambda)}$. This completes the proof.

For the Hyers-Ulam $\phi$-stability of the non-homogeneous linear differential equation (1.2), we obtain the following theorem.

Theorem 4.2 Assume that $r:[0, \infty) \rightarrow \mathbf{K}$ is a continuous function of exponential order, $\phi:[0, \infty) \rightarrow(0, \infty)$ is an increasing function and that $\lambda$ is a constant with $\Re(\lambda)>0$. The differential equation (1.2) has the Hyers-Ulam $\phi$-stability.

Proof We consider a continuously differentiable function $x:[0, \infty) \rightarrow \mathbf{K}$ of exponential order that satisfies the inequality (2.4) for all $t \geq 0$. Now we define a function $p:[0, \infty) \rightarrow$ K by $p(t):=x^{\prime}(t)+\lambda x(t)-r(t)$ for all $t \geq 0$. Then $|p(t)| \leq \phi(t) \varepsilon$ for all $t \geq 0$. It is not difficult to check

$$
\mathcal{A}\{x(t)\}=X(u)=\frac{x(0)+u P(u)+u R(u)}{u^{2}+\lambda u} .
$$

Let $y(t)=e^{-\lambda t} x(0)+\left(r(t) * e^{-\lambda t}\right)$ and we apply the Aboodh transform on both sides to get

$$
\mathcal{A}\{y(t)\}=Y(u)=\frac{x(0)+R(u)}{u^{2}+\lambda u} .
$$

On the other hand,

$$
\mathcal{A}\left\{y^{\prime}(t)+\lambda y(t)\right\}=(\lambda+u) Y(u)-\frac{x(0)}{u} .
$$

Equation (4.4) implies that $\mathcal{A}\left\{y^{\prime}(t)+\lambda y(t)\right\}=R(u)=\mathcal{A}\{r(t)\}$ and thus, $y^{\prime}(t)+\lambda y(t)=r(t)$, that is, $y(t)$ is a solution of the differential equation (1.2). Using (4.3) and (4.4), we obtain

$$
\mathcal{A}\{x(t)-y(t)\}=X(u)-Y(u)=\frac{P(u)}{\lambda+u}=u \mathcal{A}\{p(t)\} \mathcal{A}\{q(t)\},
$$


where $Q(u)=\frac{1}{u(\lambda+u)}$ which gives $q(t)=e^{-\lambda t}$. Hence $\mathcal{A}\{x(t)-y(t)\}=\mathcal{A}\{p(t) * q(t)\}$, which gives $x(t)-y(t)=p(t) * q(t)$.

Similar to the proof of Theorem 3.2, we have

$$
\begin{aligned}
|x(t)-y(t)| & =|p(t) * q(t)|=\left|\int_{0}^{t} p(s) q(t-s) d s\right| \leq \int_{0}^{t}|p(s)||q(t-s)| d s \\
& \leq \phi(t) \varepsilon e^{-\Re(\lambda) t} \int_{0}^{t} e^{\Re(\lambda) s} d s=\frac{\phi(t) \varepsilon}{\Re(\lambda)}\left(1-e^{-\Re(\lambda) t}\right) \\
& \leq K \phi(t) \varepsilon
\end{aligned}
$$

for all $t \geq 0$, where we set $K=\frac{1}{\Re(\lambda)}$. This completes the proof.

We now prove the Mittag-Leffler-Hyers-Ulam stability of the non-homogeneous linear differential equation (1.2) using the Aboodh transform method.

Theorem 4.3 Assume that $\lambda$ and $v$ are constants satisfying $\Re(\lambda)>0$ and $v>0$. Then the differential equation (1.2) has the Mittag-Leffler-Hyers-Ulam stability.

Proof Suppose that $x:[0, \infty) \rightarrow \mathbf{K}$ is a continuously differentiable function of exponential order that satisfies (2.6) for all $t \geq 0$. Consider the function $p:[0, \infty) \rightarrow \mathbf{K}$ defined by $p(t):=x^{\prime}(t)+\lambda x(t)-r(t)$ for all $t \geq 0$. Then it follows from (2.6) that $|p(t)| \leq \varepsilon E_{v}(t)$ for all $t \geq 0$.

The Aboodh transform of $p(t)$ yields the following result:

$$
P(u)=\mathcal{A}\{p(t)\}=\mathcal{A}\left\{x^{\prime}(t)+\lambda x(t)-r(t)\right\}=u X(u)-\frac{x(0)}{u}+\lambda X(u)-R(u),
$$

which further implies that

$$
X(u)=\mathcal{A}\{x(t)\}=\frac{\frac{x(0)}{u}+P(u)+R(u)}{\lambda+u} .
$$

If we set $y(t)=e^{-\lambda t} x(0)+\left(r(t) * e^{-\lambda t}\right)$ and apply the Aboodh transform on both sides of the last equality, then we obtain that

$$
Y(u)=\mathcal{A}\{y(t)\}=\frac{x(0)+u R(u)}{u^{2}+\lambda u} .
$$

On the other hand,

$$
\mathcal{A}\left\{y^{\prime}(t)+\lambda y(t)\right\}=u Y(u)-\frac{x(0)}{u}+\lambda Y(u)=(\lambda+u) Y(u)-\frac{x(0)}{u} .
$$

Then, by (4.6), we have

$$
\mathcal{A}\left\{y^{\prime}(t)+\lambda y(t)\right\}=R(u)=\mathcal{A}\{r(t)\}
$$

and thus, $y^{\prime}(t)+\lambda y(t)=r(t)$ for all $t \geq 0$. Hence $y(t)$ is a solution of the differential equation (1.2). In addition, by applying (4.5) and (4.6), we get

$$
\mathcal{A}\{x(t)-y(t)\}=\frac{P(u)}{\lambda+u}=u \mathcal{A}\{p(t)\} \mathcal{A}\{q(t)\},
$$


where $\mathcal{A}\{q(t)\}=\frac{1}{u(\lambda+u)}$, which gives $q(t)=e^{-\lambda t}$. Therefore, $\mathcal{A}\{x(t)-y(t)\}=\mathcal{A}\{p(t) * q(t)\}$ which yields $x(t)-y(t)=p(t) * q(t)$ for all $t \geq 0$. Furthermore,

$$
\begin{aligned}
|x(t)-y(t)| & =|p(t) * q(t)|=\left|\int_{0}^{t} p(s) q(t-s) d s\right| \leq \int_{0}^{t}|p(s)||q(t-s)| d s \\
& \leq \varepsilon E_{v}(t) e^{-\Re(\lambda) t} \int_{0}^{t} e^{\Re(\lambda) s} d s=\varepsilon E_{v}(t) \frac{1}{\Re(\lambda)}\left(1-e^{-\Re(\lambda) t}\right) \\
& \leq K \varepsilon E_{v}(t)
\end{aligned}
$$

for all $t \geq 0$, where we set $K=\frac{1}{\Re(\lambda)}$. This completes the proof.

Similar to the case of Theorem 4.3, the Mittag-Leffler-Hyers-Ulam $\phi$-stability of the differential equation (1.2) can be proved. For the sake of this paper's completeness, we present the whole proof.

Theorem 4.4 Assume that $\phi:[0, \infty) \rightarrow(0, \infty)$ is an increasing function and that $\lambda$ and $v$ are constants which satisfy $\Re(\lambda)>0$ and $v>0$. Then the differential equation (1.2) has the Mittag-Leffler-Hyers-Ulam $\phi$-stability.

Proof Assume that $x:[0, \infty) \rightarrow \mathbf{K}$ is a continuously differentiable function of exponential order which satisfies the inequality (2.8) for all $t \geq 0$. It is to be proved that there exist a constant $K>0$ (independent of $\varepsilon$ ) and a solution $y:[0, \infty) \rightarrow \mathbf{K}$ of the differential equation (1.2) such that

$$
|x(t)-y(t)| \leq K \phi(t) \varepsilon E_{v}(t)
$$

for all $t \geq 0$.

If we define a function $p:[0, \infty) \rightarrow \mathbf{K}$ by $p(t):=x^{\prime}(t)+\lambda x(t)-r(t)$ for all $t \geq 0$, then we have $|p(t)| \leq \phi(t) \varepsilon E_{v}(t)$ for all $t \geq 0$. By applying similar methods to Theorem 4.3, we can easily get

$$
\begin{aligned}
|x(t)-y(t)| & =\left|p(t) * e^{-\lambda t}\right|=\left|\int_{0}^{t} p(s) e^{-\lambda(t-s)} d s\right| \leq \int_{0}^{t}|p(s)|\left|e^{-\lambda(t-s)}\right| d s \\
& \leq \phi(t) \varepsilon E_{v}(t) \frac{1}{\Re(\lambda)}\left(1-e^{-\Re(\lambda) t}\right) \\
& \leq K \phi(t) \varepsilon E_{v}(t)
\end{aligned}
$$

for all $t \geq 0$, where we set $K=\frac{1}{\Re(\lambda)}$. This completes the proof.

\section{Examples}

In this section, we will introduce some examples to make it easier to understand the main results of this paper.

Example 5.1 We consider the following non-homogeneous linear differential equation:

$$
x^{\prime}(t)+5 x(t)=0 .
$$

with initial condition $x(0)=1$ and $\lambda=5$. 
If a continuously differentiable function $z:[0, \infty) \rightarrow \mathbf{K}$ of exponential order satisfies

$$
\left|z^{\prime}(t)+5 z(t)\right| \leq \varepsilon
$$

for all $t \geq 0$ and for some $\varepsilon>0$, then Theorem 3.1 implies that there exists a solution $y:[0, \infty) \rightarrow \mathbf{K}$ of the differential equation (5.1) such that

$$
|z(t)-y(t)| \leq K \varepsilon
$$

for all $t \geq 0$, where $K=\frac{1}{\Re(\lambda)}=\frac{1}{5}$. In particular, $y(t)=c e^{-5 t}$ for some constant $c \in \mathbf{K}$.

Example 5.2 We consider the following non-homogeneous linear differential equation:

$$
x^{\prime}(t)+x(t)=2 \cos t
$$

We know that $r(t)=2 \cos t$ is a function of exponential order and $\lambda=1$.

If a continuously differentiable function $z:[0, \infty) \rightarrow \mathbf{K}$ of exponential order satisfies

$$
\left|z^{\prime}(t)+z(t)-2 \cos t\right| \leq \varepsilon
$$

for all $t \geq 0$ and for some $\varepsilon>0$, then Theorem 4.1 implies that there exists a solution $y:[0, \infty) \rightarrow \mathbf{K}$ of the differential equation (5.2) such that

$$
|z(t)-y(t)| \leq K \varepsilon
$$

for all $t \geq 0$, where $K=\frac{1}{\Re(\lambda)}=1$. In particular, $y(t)=c e^{-t}+\sin t+\cos t$ for some constant $c \in \mathbf{K}$.

Example 5.3 We consider the following non-homogeneous linear differential equation:

$$
x^{\prime}(t)+3 x(t)=t,
$$

where $r(t)=t$ is a function of exponential order and $\lambda=3$.

If a continuously differentiable function $z:[0, \infty) \rightarrow \mathbf{K}$ of exponential order satisfies

$$
\left|z^{\prime}(t)+3 z(t)-t\right| \leq \varepsilon
$$

for all $t \geq 0$ and for some $\varepsilon>0$, then Theorem 4.1 implies that there exists a solution $y:[0, \infty) \rightarrow \mathbf{K}$ of the differential equation (5.3) such that

$$
|z(t)-y(t)| \leq K \varepsilon
$$

for all $t \geq 0$, where we set $K=\frac{1}{\Re(\lambda)}=\frac{1}{3}$. In particular, $y(t)=c e^{-3 t}+\frac{1}{3} t-\frac{1}{9}$ for some constant $c \in \mathbf{K}$. 


\section{Remarks}

Remark 6.1 Let $\lambda$ be a constant in $\mathbf{K}$ with $\mathfrak{R}(\lambda)>-1$. Then we have

$$
e^{-\Re(\lambda) t} \int_{0}^{t} e^{\Re(\lambda) s} d s=\frac{1}{\Re(\lambda)}\left(1-e^{-\Re(\lambda) t}\right)
$$

In the following remark, we show that there exists an integrable function $\phi:[0, \infty) \rightarrow$ $(0, \infty)$ satisfying the condition

$$
\int_{0}^{t} e^{\Re(\lambda)(t-s)} \phi(s) d s \leq K \phi(t)
$$

Remark 6.2 Let $\lambda$ be a constant in $\mathbf{K}$ with $\Re(\lambda)>-1$. If we define $\phi(t)=A e^{t}$ for all $t \geq 0$ and for some $A>0$, then we have

$$
\begin{aligned}
\int_{0}^{t} e^{\Re(\lambda)(t-s)} \phi(s) d s & =e^{-\Re(\lambda) t} \int_{0}^{t} e^{\Re(\lambda) s} A e^{s} d s \\
& =\frac{1}{1+\Re(\lambda)}\left(A e^{t}-A e^{-\Re(\lambda) t}\right) \leq \frac{1}{1+\Re(\lambda)} \phi(t) .
\end{aligned}
$$

Remark 6.3 The above examples are also true when we replace $\varepsilon$ and $K \varepsilon$ with $\phi(t) \varepsilon$ and $K \phi(t) \varepsilon$, respectively, where $\phi(t)$ is an increasing function. In this case, we see that the corresponding differential equations have the Hyers-Ulam $\phi$-stability.

Remark 6.4 The differential equations (5.2) and (5.3) have the Mittag-Leffler-HyersUlam stability if $v>0$. In particular, they also have the Mittag-Leffler-Hyers-Ulam $\phi$ stability when $\phi(t)$ is an increasing function and $v>0$.

\section{Discussion}

What results can be expected for the Hyers-Ulam stability of the differential equation (1.2) when the relevant domain is the set of all non-positive real numbers?

For any given constant $\varepsilon>0$, we consider the following inequality:

$$
\left|x^{\prime}(t)+\lambda x(t)-r(t)\right| \leq \varepsilon \quad(\text { for } t \leq 0)
$$

where $x:(-\infty, 0] \rightarrow \mathbf{K}$ is a continuously differentiable function of exponential order and $r:(-\infty, 0] \rightarrow \mathbf{K}$ is a continuous function of exponential order.

If we set $x_{1}(t)=x(-t)$ and $r_{1}(t)=r(-t)$ for all $t \geq 0$, then it follows from (7.1) that

$$
\left|x_{1}^{\prime}(t)-\lambda x_{1}(t)+r_{1}(t)\right| \leq \varepsilon \quad(\text { for } t \geq 0) .
$$

Since $x_{1}(t)$ is a continuously differentiable function of exponential order and $r_{1}(t)$ is a continuous function of exponential order, if we additionally assume that $\Re(\lambda)<0$, then Theorem 4.1 and its proof imply that there exists a continuously differentiable function $y:[0, \infty) \rightarrow \mathbf{K}$ of exponential order which satisfies

$$
y^{\prime}(t)-\lambda y(t)+r_{1}(t)=0
$$


and

$$
\left|x_{1}(t)-y(t)\right| \leq \frac{1}{|\Re(\lambda)|} \varepsilon
$$

for all $t \geq 0$.

If we define a function $z:(-\infty, 0] \rightarrow \mathbf{K}$ by $z(t)=y(-t)$ for each $t \leq 0$, then $z^{\prime}(t)=-y^{\prime}(-t)$ and so, by (7.3), we see that $z^{\prime}(t)+\lambda z(t)=-y^{\prime}(-t)+\lambda y(-t)=r(t)$ for any $t \leq 0$, i.e., $z(t)$ satisfies the differential equation (1.2) for all $t \leq 0$. Moreover, it follows from (7.4) that

$$
|x(t)-z(t)| \leq \frac{1}{|\Re(\lambda)|} \varepsilon
$$

for all $t \leq 0$.

Putting all of the above facts together, we get the following theorem.

Theorem 7.1 Assume that $r:(-\infty, 0] \rightarrow \mathbf{K}$ is a continuous function of exponential order and $\lambda$ is a constant with $\Re(\lambda)<0$. The differential equation (1.2) has the Hyers-Ulam stability for the class of all continuously differentiable functions $x:(-\infty, 0] \rightarrow \mathbf{K}$ of exponential order.

Assuming $r(t) \equiv 0$ in Theorem 7.1, we obtain the Hyers-Ulam stability of the differential equation (1.1) when the relevant domain is the set of all non-positive real numbers. Other types of Hyers-Ulam stability of the differential equation (1.2) can be similarly established when the relevant domain is the set of all non-positive real numbers.

\section{Conclusion}

In this paper, we proved the Hyers-Ulam stability, Hyers-Ulam $\phi$-stability, MittagLeffler-Hyers-Ulam stability, and Mittag-Leffler-Hyers-Ulam $\phi$-stability of the linear differential equations of first order with constant coefficients using the Aboodh transform method. In other words, we established sufficient criteria for the Hyers-Ulam stability of first-order linear differential equations with constant coefficients using the Aboodh transform method.

Moreover, we provided a new method to investigate the Hyers-Ulam stability of differential equations. This is the first attempt to use the Aboodh transform to prove the Hyers-Ulam stability for linear differential equations of the first order. Furthermore, we showed that the Aboodh transform method is more convenient for investigating the stability problems for linear differential equations with constant coefficients.

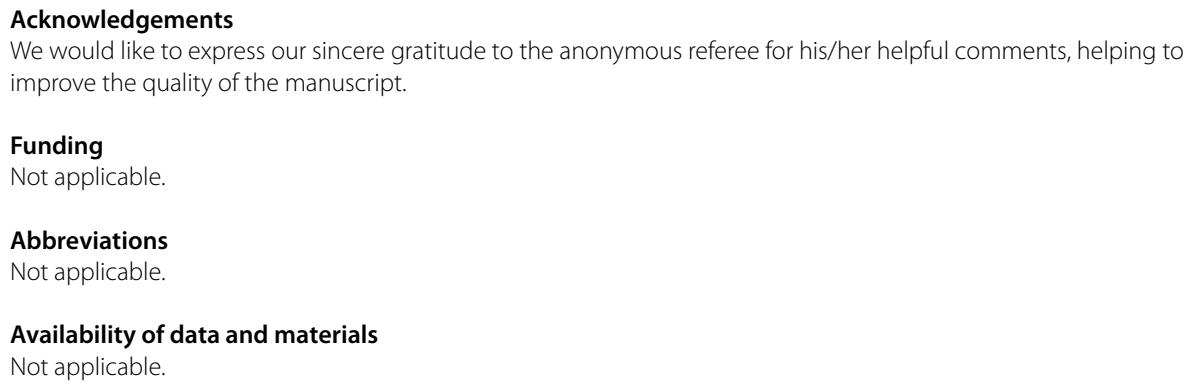


Ethics approval and consent to participate

Not applicable.

\section{Competing interests}

The authors declare that they have no competing interests.

\section{Consent for publication}

Not applicable.

\section{Authors' contributions}

The authors equally conceived of the study, participated in its design and coordination, drafted the manuscript, participated in the sequence alignment, and read and approved the final manuscript.

\section{Author details}

'PG and Research Department of Mathematics, Sacred Heart College (Autonomous, 635601 Tirupattur, India.

${ }^{2}$ Department of Mathematics, Sri Sai Ram Institute of Technology, 600044 Chennai, India. ${ }^{3}$ Research Institute for Natural Sciences, Hanyang University, 04763 Seoul, Korea. ${ }^{4}$ Department of Data Science, Daejin University, 11159 Kyunggi, Korea.

\section{Publisher's Note}

Springer Nature remains neutral with regard to jurisdictional claims in published maps and institutional affiliations.

Received: 10 April 2021 Accepted: 14 July 2021 Published online: 29 July 2021

\section{References}

1. Ulam, S.M.: Problem in Modern Mathematics. Willey, New York (1960)

2. Hyers, D.H.: On the stability of a linear functional equation. Proc. Natl. Acad. Sci. USA 27, $222-224$ (1941)

3. Aoki, T.: On the stability of the linear transformation in Banach spaces. J. Math. Soc. Jpn. 2, 64-66 (1950)

4. Bourgin, D.G.: Classes of transformations and bordering transformations. Bull. Am. Math. Soc. 57, 223-237 (1951)

5. Rassias, J.M.: On approximately of approximately linear mappings by linear mappings. J. Funct. Anal. 46, 126-130 (1982)

6. Rassias, T.M.: On the stability of the linear mappings in Banach spaces. Proc. Am. Math. Soc. 72, $297-300$ (1978)

7. Vaezi, H.: Hyers-Ulam stability of weighted composition operators on disc algebra. Int. J. Math. Comput. 10, 150-154 (2011)

8. Obłoza, M.: Hyers stability of the linear differential equation. Rocznik Nauk.-Dydakt. Prace Mat. 13, $259-270$ (1993)

9. Obłoza, M.: Connections between Hyers and Lyapunov stability of the ordinary differential equations. Rocznik Nauk.-Dydakt. Prace Mat. 14, 141-146 (1997)

10. Alsina, C., Ger, R.: On some inequalities and stability results related to the exponential function. J. Inequal. Appl. 2, 373-380 (1998)

11. Takahasi, S.E., Miura, T., Miyajima, S.: On the Hyers-Ulam stability of the Banach space-valued differential equation $y^{\prime}=\alpha y$. Bull. Korean Math. Soc. 39, 309-315 (2002)

12. Jung, S.: Hyers-Ulam stability of linear differential equation of first order. Appl. Math. Lett. 17, 1135-1140 (2004)

13. Jung, S.: Hyers-Ulam stability of linear differential equations of first order (III). J. Math. Anal. Appl. 311, 139-146 (2005)

14. Jung, S.: Hyers-Ulam stability of linear differential equations of first order (II). Appl. Math. Lett. 19, 854-858 (2006)

15. Jung, S.: Hyers-Ulam stability of a system of first order linear differential equations with constant coefficients. J. Math. Anal. Appl. 320, 549-561 (2006)

16. Miura, T:: On the Hyers-Ulam stability of a differentiable map. Sci. Math. Japan 55, 17-24 (2002)

17. Miura, T., Takahasi, S.E., Choda, H.: On the Hyers-Ulam stability of real continuous function valued differentiable map $y^{\prime}=\lambda y$. Tokyo J. Math. 24, 467-476 (2001)

18. Miura, T., Jung, S., Takahasi, S.E.: Hyers-Ulam-Rassias stability of the Banach space valued linear differential equations. J. Korean Math. Soc. 41, 995-1005 (2004)

19. Wang, G., Zhou, M., Sun, L.: Hyers-Ulam stability of linear differential equations of first order. Appl. Math. Lett. 21, 1024-1028 (2008)

20. Rus, I.A.: Ulam stabilities of ordinary differential equations in a Banach space. Carpath. J. Math. 26, 103-107 (2010)

21. Alqifiary, Q.H., Jung, S.: Laplace transform and generalized Hyers-Ulam stability of differential equations. Electron. J. Differ. Equ. 2014, Article ID 80 (2014)

22. Rezaei, H., Jung, S., Rassias, T.M.: Laplace transform and Hyers-Ulam stability of linear differential equations. J. Math. Anal. Appl. 403, 244-251 (2013)

23. Cimpean, D.S., Popa, D.: On the stability of the linear differential equation of higher order with constant coefficients Appl. Math. Comput. 217, 4141-4146 (2010)

24. Buakird, A., Saejung, S.: Ulam stability with respect to a directed graph for some fixed point equations. Carpath. J. Math. 35, 23-30 (2019)

25. Fukutaka, R., Onitsuka, M.: Best constant in Hyers-Ulam stability of first-order homogeneous linear differential equations with a periodic coefficient. J. Math. Anal. Appl. 473, 1432-1446 (2019)

26. Huang, J., Jung, S., Li, Y.: On Hyers-Ulam stability of nonlinear differential equations. Bull. Korean Math. Soc. 52 , 685-697 (2015)

27. Li, T., Zada, A., Faisal, S.: Hyers-Ulam stability of $n$th order linear differential equations. J. Nonlinear Sci. Appl. 9 2070-2075 (2016)

28. Li, Y., Shen, Y.: Hyers-Ulam stability of linear differential equations of second order. Appl. Math. Lett. 23, 306-309 (2010)

29. Murali, R., Bodaghi, A., Selvan, A.P.: Stability for the third order linear ordinary differential equation. Int. J. Math. Comput. 30, 87-92 (2019) 
30. Murali, R., Selvan, A.P.: On the generalized Hyers-Ulam stability of linear ordinary differential equations of higher order. Int. J. Pure Appl. Math. 117(12), 317-326 (2017)

31. Murali, R., Selvan, A.P.: Hyers-Ulam-Rassias stability for the linear ordinary differential equation of third order. Kragujev. J. Math. 42, 579-590 (2018)

32. Murali, R., Selvan, A.P.: Ulam stability of third order linear differential equations. Int. J. Pure Appl. Math. 120(9), 217-225 (2018)

33. Murali, R., Selvan, A.P.: Hyers-Ulam stability of $n$th order linear differential equation. Proyecciones 38(3), 553-566 (2019)

34. Murali, R., Selvan, A.P.: Hyers-Ulam stability of a free and forced vibrations. Kragujev. J. Math. 44(2), 299-312 (2020)

35. Murali, R., Park, C., Selvan, A.P.: Hyers-Ulam stability for an nth order differential equation using fixed point approach. J. Appl. Anal. Comput. 11, 614-631 (2021)

36. Onitsuka, M.: Hyers-Ulam stability of first order linear differential equations of Caratheodory type and its application. Appl. Math. Lett. 90, 61-68 (2019)

37. Onitsuka, M., Shoji, T.: Hyers-Ulam stability of first order homogeneous linear differential equations with a real valued coefficients. Appl. Math. Lett. 63, 102-108 (2017)

38. Murali, R., Selvan, A.P., Park, C.: Ulam stability of linear differential equations using Fourier transform. AlMS Math. 5, 766-780 (2019)

39. Murali, R., Selvan, A.P.: Fourier transforms and Ulam stabilities of linear differential equations. In: Front. Funct. Equ. Anal. Inequal., pp. 195-217. Springer, Switzerland (2019)

40. Rassias, J.M., Murali, R., Selvan, A.P.: Mittag-Leffler-Hyers-Ulam stability of linear differential equations using Fourier transforms. J. Comput. Anal. Appl. 29, 68-85 (2021)

41. Murali, R., Selvan, A.P.: Mittag-Leffler-Hyers-Ulam stability of a linear differential equations of first order using Laplace transforms. Canad. J. Appl. Math. 2(2), 47-59 (2020)

42. Aboodh, K.S.: The new integral transform "Aboodh transform". Glob. J. Pure Appl. Math. 9, 35-43 (2013)

43. Aboodh, K.S.: Application of new transform "Aboodh transform" to partial differential equations. Glob. J. Pure Appl. Math. 10, 249-254 (2014)

44. Alshikh, A.A., Mahgob, M.M.A.: A comparative study between Laplace transform and two new integrals "Elzaki" transform and Aboodh transform. Pure Appl. Math. 5(5), 145-150 (2016)

45. Aboodh, K.S.: Solving porous medium equation using Aboodh transform homotopy perturbation method. Pure Appl. Math. J. 4(6), 271-276 (2016)

46. Aggarwal, S., Sharma, N., Chauhan, R.: Solution of linear Volterra integro-differential equations of second kind using Mahgoub transform. Int. J. Latest Tech. Eng. Manag. Appl. Sci. VII(V), 173-176 (2018)

47. Osu, B.O., Sampson, V.U.: Application of Aboodh transform to the solution of stochastic differential equation. J. Adv. Research Appl. Math. Stat. 3(4), 12-18 (2018)

48. Kalvandi, V., Eghbali, N., Rassias, J.M.: Mittag-Leffler-Hyers-Ulam stability of fractional differential equations of second order. J. Math. Ext. 13(1), 1-15 (2019)

\section{Submit your manuscript to a SpringerOpen ${ }^{\circ}$ journal and benefit from:}

- Convenient online submission

- Rigorous peer review

- Open access: articles freely available online

- High visibility within the field

- Retaining the copyright to your article

Submit your next manuscript at $\boldsymbol{\nabla}$ springeropen.com 